\title{
Laterally filtered 1D inversions of small-loop, frequency-domain EMI data from a chemical waste site
}

\author{
Patricia Martinelli ${ }^{1}$ and María Celeste Duplaá ${ }^{2}$
}

\begin{abstract}
We made a study in the backyard of an agrochemical plant using a small-loop, frequency-domain electromagnetic induction (EMI) system. Such systems are very sensitive to conductive structures buried at shallow depths. Frequently, they are only used to locate and delimit these structures by direct observation of data. However, much more information can be obtained by applying numerical modeling techniques to the data. First we mapped an anomalous zone that indicates the possible presence of buried waste or some other underground contamination by visualizing data. Then we applied a 1D inversion method to the data from this zone. By joining 1D inversion results, this method builds 2D images of the subsoil structure below survey lines.
\end{abstract}

Because the code applies smoothness constraints to the 1D inversions, the subsoil properties in these 2D images change gradually with depth. The code does not impose any correlation between the data or 1D models corresponding to neighboring points, so sharp lateral changes can appear. Several of them do not represent real features of the subsoil. We designed and applied two spatial filters to smooth the spurious lateral variations in our models. One correlates the data acquired at adjacent points prior to inversions. The other applies an analogous correlation to the inverse models obtained from the original data. Both filters greatly improve the quality of the 2D images. Compiling these results, we obtained a 3D model of the subsoil that characterizes the anomalous structure. Excavations made later at the site confirmed the results.

\section{INTRODUCTION}

Small-loop frequency-domain electromagnetic induction (SLEM) systems (McNeill, 1980; Won et al., 1996; McNeill and Bosnar, 1999; Won, 2003) often are used for unexploded ordnance (UXO) detection (Bell et al., 2001; Miller et al., 2001; Won et al., 2001; Butler, 2004), environmental applications such as contaminant detection or waste-site exploration (Tezkan, 1999; Hendrickx et al., 2002; Eigenberg and Nienaber, 2003; Auken et al., 2006), and archaeological prospecting (Witten et al., 2003; Osella et al., 2005; Lascano et al., 2006) because they are very sensitive to the presence of structures buried at shallow depths, especially conductive ones. These systems are formed by two small coils, a transmitter and a receiver, that usually are coplanar. The coils are separated at a fixed distance and are moved over the surface of the ground at an approximately constant height. The transmitter generates a controlled, primary magnetic field, and the instrument determines the in-phase and quadrature components of the secondary field detected at the receiver, which are expressed in parts per million ( $\mathrm{ppm}$ ) against the primary field. These components contain information about the electrical conductivity of the subsoil. In addition, the in-phase component also depends on magnetic susceptibility (Won et al., 1998; Huang and Won, 2000).

One of the main advantages of SLEM systems is that they do not require direct contact with the ground and therefore are much faster than geoelectric or other electromagnetic induction (EMI) methods such as audiomagnetotellurics (AMT). This is particularly important for shallow prospecting because the surveyed areas usually are large compared with the required lateral resolution.

The use of 2D inversion methods to interpret geoelectric and AMT data is a common approach. Sometimes, 3D forward and inverse modeling methods also are applied, although in those cases, much more extended and time-consuming fieldwork is required. On

Manuscript received by the Editor 30 May 2007; revised manuscript received 29 December 2007; published online 23 May 2008.

${ }^{1}$ CONICET (Consejo Nacional de Investigaciones Científicas y Técnicas), Argentina, and Universidad de Buenos Aires. Departamento de Física, Facultad de Ciencias Exactas y Naturales, Buenos Aires, Argentina. E-mail: pmartine@ df.uba.ar.

${ }^{2}$ ANPCyT (Agencia Nacional de Promoción Científica Tecnológica), Argentina, and Universidad de Buenos Aires. Departamento de Física, Facultad de Ciencias Exactas y Naturales, Buenos Aires, Argentina. E-mail: duplaa@df.uba.ar. (c) 2008 Society of Exploration Geophysicists. All rights reserved. 
the contrary, SLEM data still are interpreted qualitatively only by direct observation of the plan views of the in-phase and quadrature components measured at each frequency. This detects anomalous structures and approximately maps their lateral extension but does not permit quantitative depth characterization. Numerical modeling techniques seldom are used - not because of the difficulty of performing necessary fieldwork (SLEM data collection is rapid) but because of the inherent difficulty of accurately calculating the inductive response of the ground to $3 \mathrm{D}$, highly localized magnetic dipole sources in useful computation times.

This is a particularly complex problem, so there are relatively few numerical methods to interpret SLEM data and they are not applied routinely. The methods proposed to calculate the response of metallic bodies such as UXO generally assume these objects are in free space (Geng et al., 1999; Ao et al., 2002; Shubitidze et al., 2002; Sun et al., 2004). This is because metals are orders of magnitude more conductive than common background media. On the other hand, for environmental or archaeological applications, the background must be considered along with the targets. Several forward and inverse methods that consider general 3D subsoil models have been developed (Newman and Alumbaugh, 1995, 1997; Pérez-Flores et al., 2001; Sasaki, 2001; Haber et al., 2004), but their use for modeling realistic structures implies a high computing cost. This cost is lower for 2D subsoil models; however, this subject has received little attention in the literature.

A few 2D methods have been reported for electric dipole sources (Unsworth et al., 1993; Mitsuhata, 2000; Mitsuhata et al., 2002). Mitsuhata (2000) considers also magnetic dipole sources. Martinelli et al. (2006) present a forward method for calculating the response of 2D multilayered structures to arbitrarily oriented magnetic dipoles using a Rayleigh-fast Fourier transform technique. Lascano et al. (2006) use it to interpret SLEM data from an archaeological site. In

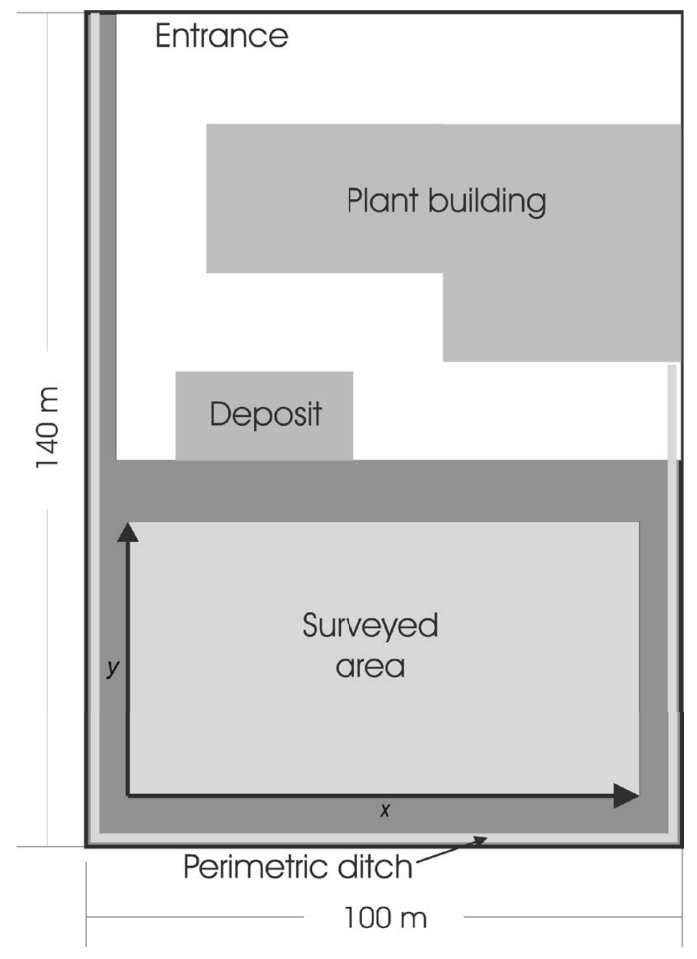

Figure 1. Schematic of the agrochemical plant and the surveyed area. addition, Sasaki and Meju (2006) present a 2D inversion method for horizontal transmitting and receiving coils. Their method uses staggered finite differences for the forward modeling and, during the inversion, smooths models by minimizing a combination of the level of misfit and the amount of structure in the models. Sasaki and Meju (2006) apply it to invert synthetic data and to image water-bearing targets in a granitic terrain, obtaining very good results.

One-dimensional inversion methods are very fast today - so much so that their application has become very practical, even for interpreting large amounts of data. This is the most frequent case in SLEM studies considering the rapidity of these systems. Farquharson et al. (2003) propose a 1D inversion method for horizontally or vertically oriented coils. Their method uses the matrix-propagation approach to calculate forward responses and applies smoothness constraints similar to the method of Sasaki and Meju (2006) during inversions.

By combining the results of 1D inversions, 2D models of the subsoil along survey lines and 3D models of the subsoil in a whole study area can be obtained. No lateral smoothness constraints are applied, so sharp lateral variations are freely allowed to appear in these models - and they often do. Some denote real changes in the subsoil structure, others arise from 2D or 3D effects, and yet others are artifacts associated with data noise. As an approach to solving this problem, Monteiro Santos (2004) proposes a laterally constrained inversion (LCI) scheme. This method combines 1D inversions with 2D smoothness constraints between adjacent 1D models obtained along acquisition lines, and it can interpret data acquired with single-frequency EMI systems. Auken et al. (2005) propose a similar approach for interpreting DC resistivity data.

Taking into account the feasibility of 1D inversion methods, it is important to explore in which cases they can provide acceptable approximations of the actual subsoil structures — particularly when $2 \mathrm{D}$ or $3 \mathrm{D}$ anomalies are present — and to investigate possible procedures to improve the resulting models. A necessary part of the analysis involves testing the techniques through their application to interpret field data from diverse geologic structures.

In 2006, we performed an SLEM survey in the backyard of an agrochemical plant to obtain information that could be useful for later remediation work. First we identified an area with anomalously increased responses. Next we obtained an electrical image of the subsoil in that zone by applying the 1D inversion method developed by Farquharson et al. (2003). This provided a first characterization of the conductive structure responsible for the observed anomaly. However, the images obtained for all of the lines contained many spurious lateral jumps in conductivity from point to point. To reduce these jumps, we developed and applied two distinct horizontal smoothing filters. One correlated data acquired at neighboring points, prior to inversions. The other directly correlated the 1D inverse models obtained from the original data. Each method significantly improved the quality of the inverted resistivity model and, accordingly, the visualization of the conductive anomaly. Here, we present our results.

\section{DATA ACQUISITION AND QUALITATIVE INTERPRETATION}

The prospect zone was in the backyard of the agrochemical plant (Figure 1). The soil presented an almost flat topography, with the exception of a perimetric ditch, and was covered only by slight grass. 
Prior to the survey, the machinery was moved to the front area and visible objects lying on the ground were removed.

We used a portable GEM-2 SLEM instrument (Won et al., 1996), manufactured by Geophex Limited, to collect the data. In this system, the coils are coplanar and are separated by $1.66 \mathrm{~m}$. We acquired data at six frequencies between 2575 and 47,025 Hz along 24 parallel lines, oriented in the $x$-direction and spaced $2 \mathrm{~m}$ apart. At each line, we performed 87 soundings, spaced $1 \mathrm{~m}$ apart. To decrease error level, we set the instrument to take 10 measurements for each frequency at each sounding point; then we calculated the arithmetic mean of each sample. Dipole axes were oriented in the vertical direction $z$ and instrument axis was parallel to prospection lines.

Figures 2 and 3 show, respectively, the plan views of the in-phase and quadrature components, in parts per million relative to the primary field. In the area measuring approximately $0 \leq x \leq 20 \mathrm{~m}$ and $10 \leq y \leq 20 \mathrm{~m}$ there was an important increase of the in-phase response at all frequencies and an increase of the quadrature response at frequencies lower than $13,575 \mathrm{~Hz}$. This indicated the presence of a structure with anomalously high electrical conductivity, which could correspond to buried waste or some kind of underground contamination. To obtain more quantitative information about this anomaly, we numerically modeled the data corresponding to the lines located between $y=4$ and $y=26 \mathrm{~m}$.

\section{D INVERSIONS}

To the data acquired along those lines, we applied the EM1DFM inversion code developed by the Geophysical Inversion Facility of the University of British Columbia (UBC-GIF), based on work by Farquharson et al. (2003). This method fits the SLEM response recorded at each sounding point as a function of frequency, using 1D models of the electrical conductivity and/or magnetic susceptibility of the subsoil that are subject to smoothness constraints. Then it generates $2 \mathrm{D}$ images of the subsoil structure below each survey line by joining the 1D inverse models obtained along that line. Parallel 2D profiles can be combined with graphical software to obtain a 3D view of the subsoil in the entire study area.

In the 2D models obtained as described, subsoil properties vary gradually with depth but generally do not vary gradually in the later-
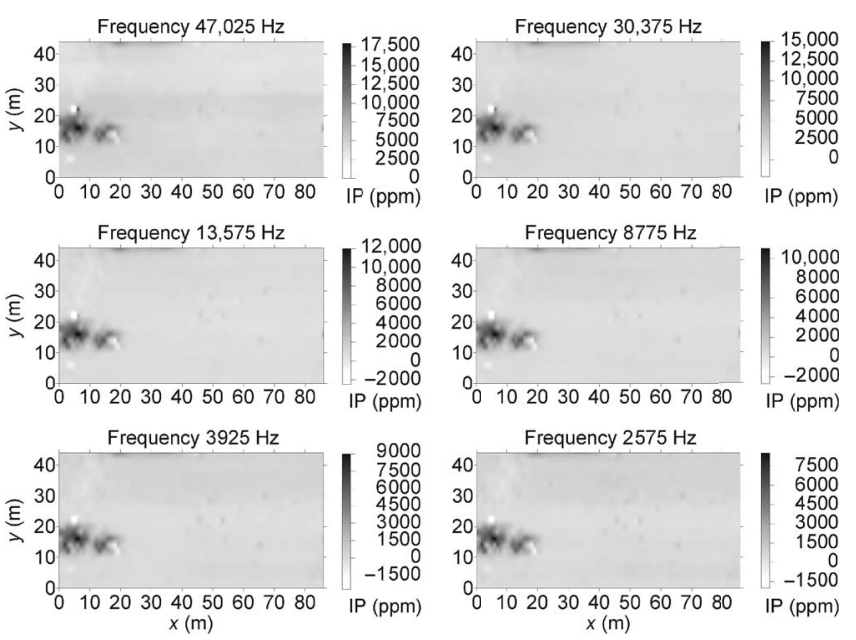

Figure 2. Plan views of the in-phase component at the measured frequencies, in parts per million relative to the primary field (IP $=$ inphase). al direction. The program does not impose a correlation between the data to be inverted corresponding to neighboring points or between the obtained 1D inverse models, so sharp lateral changes can appear freely. As stated, many of them do not correspond to real features of the subsoil.

We inverted both components together and each one separately. For all of the lines, the inversions of the quadrature component alone provided the best results, which corresponded to the lowest misfit levels, probably because this component was less noisy than the inphase component. To invert the quadrature component, we considered only the electrical conductivity of the subsoil because the sensitivity of this component to the magnetic susceptibility is low. Figure 4 shows the resulting models obtained at four representative lines, and Figure 5 is a 2D plot of the misfit for this inversion. The misfit $\phi_{i j}$ at each sounding point was calculated as the standard rms error:
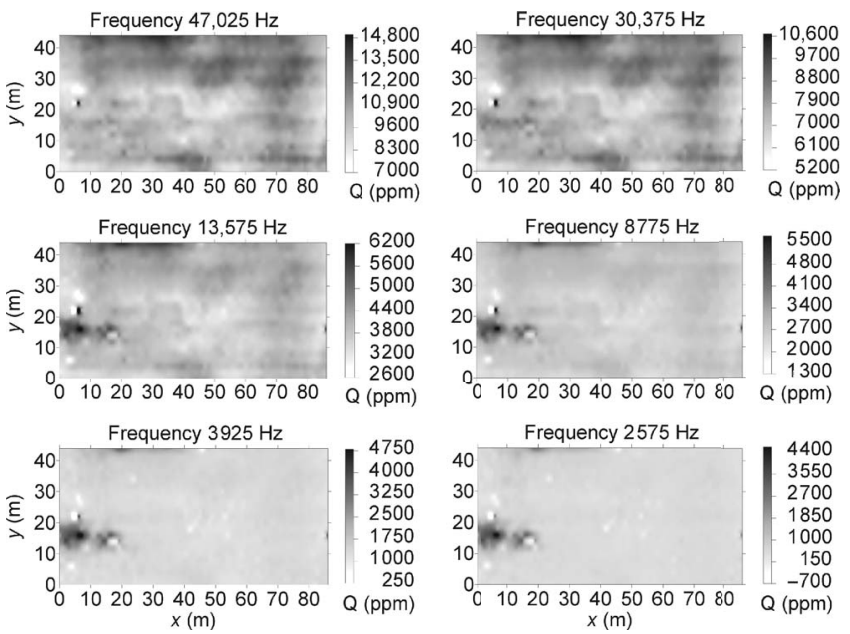

Figure 3. Plan views of the quadrature component at the measured frequencies, in parts per million relative to the primary field $(\mathrm{Q}=$ quadrature).

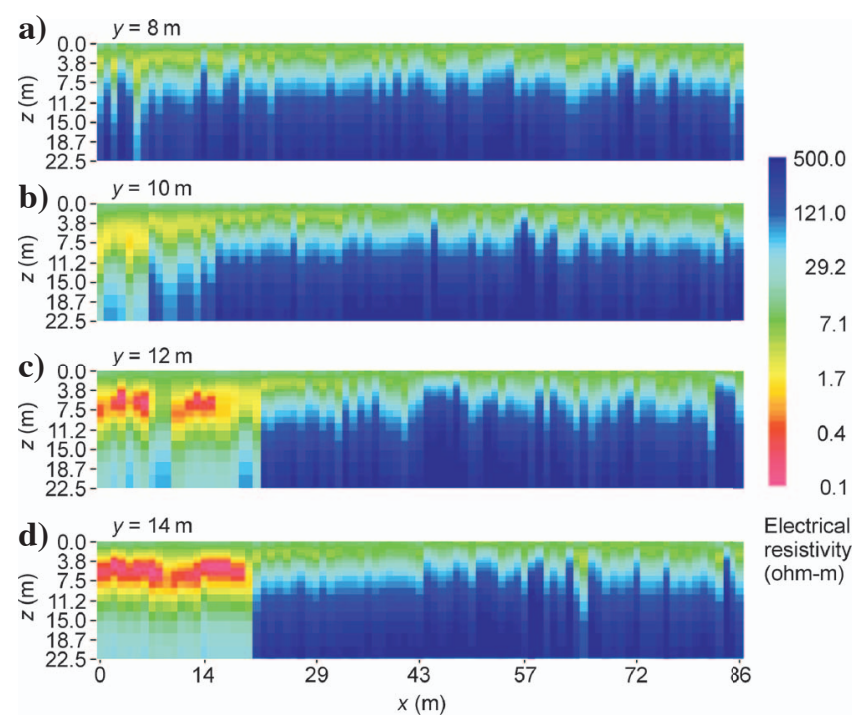

Figure 4. Electrical models of the subsoil along the lines (a) $y$ $=8 \mathrm{~m}$, (b) $y=10 \mathrm{~m}$, (c) $y=12 \mathrm{~m}$, and (d) $y=14 \mathrm{~m}$, obtained by applying the EM1DFM 1D inversion method to the measured quadrature components. 


$$
\phi_{i j}=\sqrt{\frac{1}{N_{\nu}} \sum_{k=1}^{N_{\nu}}\left(\frac{Q_{i, j, k}-Q_{i, j, k}^{P}}{s_{i j k}}\right)^{2}},
$$

where $Q_{i, j, k}$ and $Q_{i, j, k}^{P}$ are, respectively, the observed and predicted values of the quadrature component at point $\left(x_{i}, y_{j}\right)$ and frequency $k$. The value $N_{\nu}$ is the number of measured frequencies, and $s_{i j k}$ is the standard deviation of each data point.

One-dimensional inversions detected the conductive anomaly and provided a first characterization of its electrical structure. Even the top and bottom of the more conductive sector were delimited roughly. Nevertheless, the models obtained for all of the lines exhibited numerous short-scale lateral conductivity variations. Many of those variations were correlated to lateral variability of the quadrature component as a result of data noise. The changes from point to point in the attained misfit and in the error level of each data set also increased the lateral variability of the models. In addition, comparatively high error levels were found in the anomalous zone, which could result partly from the presence of some 3D effects. There, misfits well below one were obtained. This indicated a particularly important overfitting of noise. In Figure 6, we show, as a representative example, the data and predicted model responses for the line $y$ $=12 \mathrm{~m}$, along with the corresponding misfit. Comparing Figure $4 \mathrm{c}$ with Figure 6, we see the undesired effects become strikingly evident.

To obtain the selected final model, we varied the parameters that control the inversions until we could reduce many of the initial lateral changes. The inversion method provides three automatic and one manual procedure to determine the value of the trade-off parameter $\beta$ at each inversion point. This parameter controls the balance between the model misfit and model norm (see Farquharson et al. (2003) for details). When the automatic procedures are used, the resulting values of $\beta$ and, accordingly, the misfits might have large variations from point to point. On the other hand, in the manual procedure, the user supplies the value of $\beta$, which is the same for all points. In our case, this procedure gave much better results than the

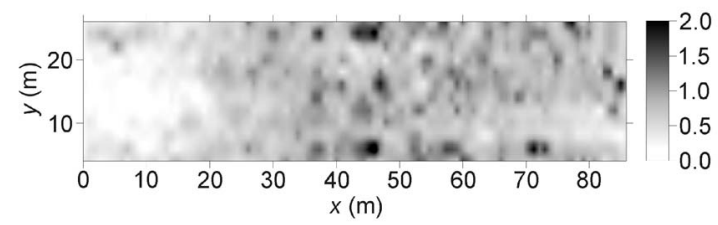

Figure 5. A2D plot of the misfit between the measured and predicted quadrature components for the model in Figure 4.

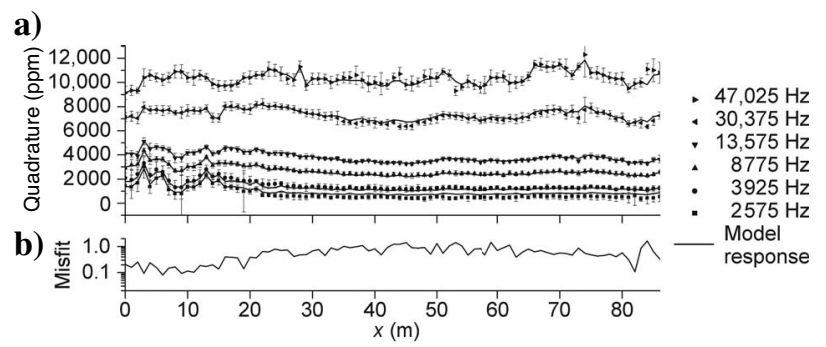

Figure 6. (a) Quadrature data measured along the line $y=12 \mathrm{~m}$, together with the forward response of the inverse model shown in Figure 4c. (b) Plot of the corresponding misfit for that line. automatic ones because it reduced misfit variations along the lines.

By increasing the supplied value of $\beta$, lateral changes generally decrease but vertical smoothing increases. Thus the user can control the relative weight of each factor in the $2 \mathrm{D}$ models. The results shown in Figure 4 were obtained with $\beta=1000$ for default values of the parameters $\alpha_{s}^{\sigma}, \alpha_{z}^{\sigma}$, and $\tau$, equal to $0.01,1$, and 0.01 , respectively. The initial model was a half-space of $600 \mathrm{ohm}-\mathrm{m}$ resistivity, equal to the expected resistivity of the deepest layers. The mean value of the misfit for this inversion was 0.69 , somewhat lower than one. Considering this, we tried to increase $\beta$ further, but this reduced the vertical resolution much more than the lateral changes.

\section{LATERAL FILTERING}

We developed two spatial filters to reduce the remaining unrealistic lateral variations occurring in the $2 \mathrm{D}$ models without significantly increasing vertical smoothing. Both can be applied only once or iteratively several times. For their formulation, we assume that survey lines are parallel to the $x$-direction and are spaced a constant distance $\Delta y$. The points along each line also are spaced equally, and their spacing $\Delta x$ is the same for all lines. In our case, $\Delta x=1 \mathrm{~m}$ and $\Delta y$ $=2 \mathrm{~m}$.

\section{Preinversion data-smoothing filter}

The first lateral filter correlates the quadrature components from neighboring points before inversions. It replaces the quadrature component of the sounding point $\left(x_{i}, y_{j}\right)$ at the frequency $\nu_{k}$ after $l$ applications of the filter $Q_{i, j, k}^{(l)}$ by the following weighted average:

$$
Q_{i, j, k}^{(l+1)}=\left[\sum_{n, m \in\left[r_{n m} \leq R\right]} \frac{Q_{i+n, j+m, k}^{(l)}}{A^{\frac{r_{n m}}{\Delta x}}}\right]\left[\sum_{n, m \in\left[r_{n m} \leq R\right]} A^{\frac{r_{n m}}{\Delta x}}\right]^{-1},
$$

where $Q_{i, j, k}^{(0)}$ are the measured quadrature components; $r_{n m}$ $=\sqrt{(n \Delta x)^{2}+(m \Delta y)^{2}}$ is the distance between points $\left(x_{i}, y_{j}\right)$ and $\left(x_{i+n}, y_{j+m}\right)$ ( $n$ and $m$ are integers); $R$ is the radius of the region around the point $\left(x_{i}, y_{j}\right)$ considered for the smoothing, typically a natural number $N$ times $\Delta x$; and $A$ is a real number greater than one that controls the velocity at which the relative weight of the neighbors decreases when $r_{n m}$ increases.

This filter is very versatile. By varying the parameters $R$ (or $N$ ) and $A$ along with the number of successive applications, the user can control the smoothing process and the lateral changes allowed in the final model. The best choices will depend on the characteristics of the lateral variability of data for each particular situation and the misfit levels successively attained. In our case, we obtained very good results with $N=3$ and $A=2$, applying the filter two or three times. This smoothed almost all lateral variations considered to be spurious, along all lines, and greatly improved visualization of the target structure. The mean values of misfit obtained after the first, second, and third applications of this filter were, respectively, 0.77 , 0.85 , and 0.88 .

Figure 7 shows the corresponding inverse models obtained for the line $y=12 \mathrm{~m}$. The successive enhancements are evident. Figure $8 \mathrm{a}$ compares the responses of these models to the data and to the response of the model obtained without lateral filtering (shown in Figure $4 \mathrm{c}$ ). Figure $8 \mathrm{~b}$ shows the corresponding misfits along that line. Many of the short-scale lateral variations in the responses attributable to the noise, especially those occurring just over the anomalous 
zone, were gradually reduced and the general behavior of data was followed. As mentioned, the misfit attained over the anomaly without filtering was much lower than one (about 0.17), which implied that much of the noise was fitted together with the relevant features of data. After lateral filtering, the mean values of the misfit in that zone increased to, respectively, $0.50,0.70$, and 0.82 as long as the fitting of noise decreased.

The filtering process is extremely fast. Each application of the filter to our data set demanded less than $5 \mathrm{~s}$ in a Pentium dual-core, 2.8 -GHz personal computer. In addition, the filtering process is independent of the 1D inversion method because it is applied directly to the data. A final important advantage of this filter is its high stability. We applied it six times, and the anomaly still could be identified clearly (Figure 9).

\section{Postinversion model-smoothing filter}

The second lateral filter is analogous to the preinversion datasmoothing filter, but it is applied directly to the inverse models. If $\sigma_{i, j, k}^{(l)}$ is the electrical conductivity of the layer $k$ below the point $\left(x_{i}, y_{j}\right)$ after applying this filter $l$ times, then

\section{a)}

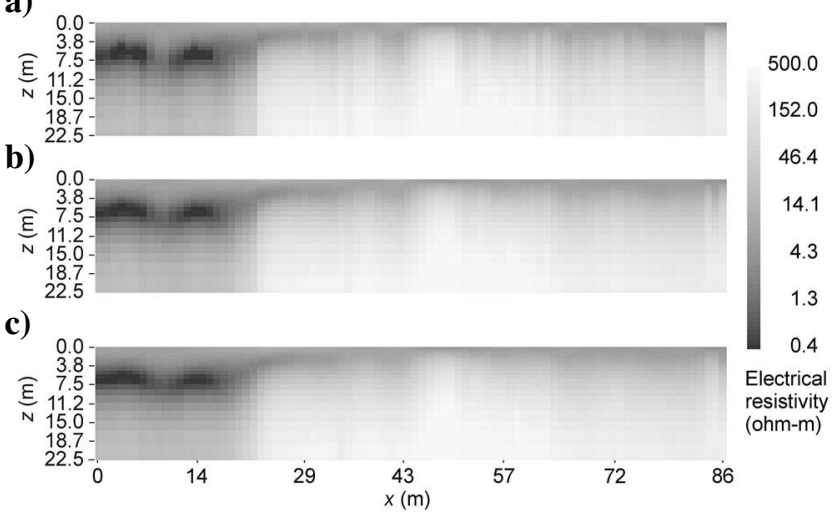

Figure 7. Models obtained for the line $y=12 \mathrm{~m}$ applying the EM1DFM 1D inversion code to the quadrature components filtered (a) one, (b) two, and (c) three times, using the preinversion datasmoothing filter defined by equation 2 with $N=3$ and $A=2$.

a)

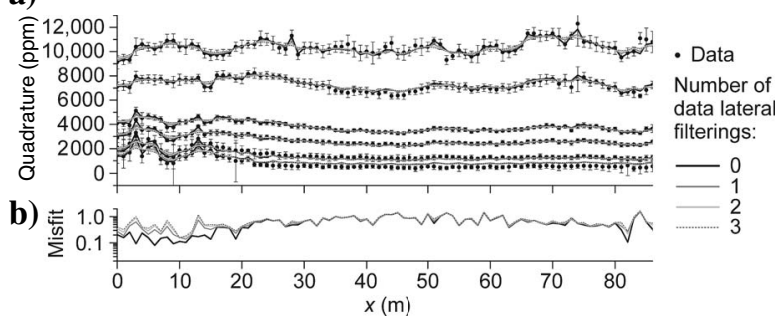

Figure 8. (a) Quadrature data measured along the line $y=12$, along with responses of the inverse models obtained without filtering data (Figure 4c) and applying the preinversion data-smoothing filter $(N$ $=3$ and $A=2$ ) one, two, and three times (models shown in Figure 7a-c, respectively). (b) Plot of the corresponding misfits.

$$
\sigma_{i, j, k}^{(l+1)}=\left[\sum_{n, m \in\left[r_{n m} \leq R\right]} \frac{\sigma_{i+n, j+m, k}^{(l)}}{A^{\frac{r_{n m}}{\Delta x}}}\right]\left[\sum_{n, m \in\left[r_{n m} \leq R\right]} A^{\frac{r_{n m}}{\Delta x}}\right]^{-1},
$$

where $\sigma_{i, j, k}^{(0)}$ are the conductivities of the inverse model obtained from the original quadrature data. The rest of the parameters have the same meaning as in the former case.

We applied this postinversion model-smoothing filter one, two, and three times, again with $N=3$ and $A=2$. The mean values of misfit attained - 0.80,0.88, and 0.92 , respectively - were only somewhat greater than the ones obtained with the preinversion filter. Figure 10 shows the models obtained for $y=12 \mathrm{~m}$ after each application of this filter. Comparing Figures 7 and 10, one can appreciate that the smoothing rate of this filter is a bit greater. Nevertheless, both provide very good results and acceptable misfits at almost all points.

\section{FINAL MODEL}

Figure 11 shows the final images of the subsoil along lines $y$ $=8 \mathrm{~m}$ to $y=22 \mathrm{~m}$, which covered the whole anomalous region. We obtained these images by applying the preinversion datasmoothing filter two times, with $N=3$ and $A=2$. The misfit for this inversion is shown in Figure 12. Its mean value is 0.85 . Comparing Figures 4 and 11 reveals the benefits of our proposed lateral filtering schemes. In this final model, the structure of the anomaly can

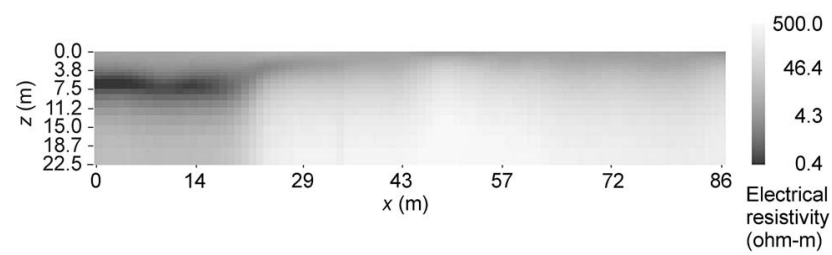

Figure 9. Model obtained along the line $y=12 \mathrm{~m}$, applying the EM1DFM 1D inversion code to the quadrature components filtered six times, using the filter defined by equation 2 with $N=3$ and $A$ $=2$.

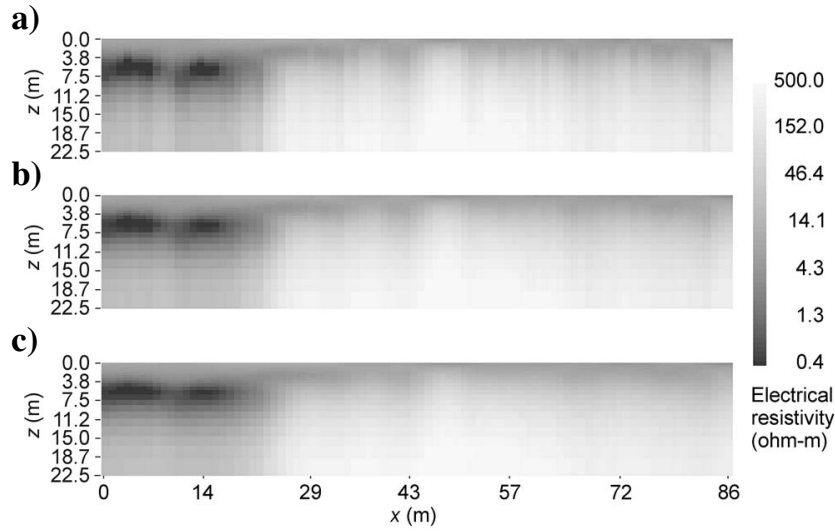

Figure 10. Models obtained after applying to the model shown in Figure $4 \mathrm{c}$ the postinversion model-smoothing filter, defined by equation 3 with $N=3$ and $A=2$, (a) one, (b) two, and (c) three times. 
be appreciated more clearly, including the existence of two separate volumes with resistivity lower than $0.27 \mathrm{ohm}-\mathrm{m}$.

From the excavations performed later at the site during remediation work, we corroborated that our combination of lateral filtering and $1 \mathrm{D}$ inversions not only could detect but also could provide a very adequate characterization of the anomalous structure. Hundreds of metallic barrels were found, containing production remainders ranging from resistive to moderately conductive. These barrels were buried between 3-4 and 7.5-8 $\mathrm{m}$ deep in two almost rectangular zones,

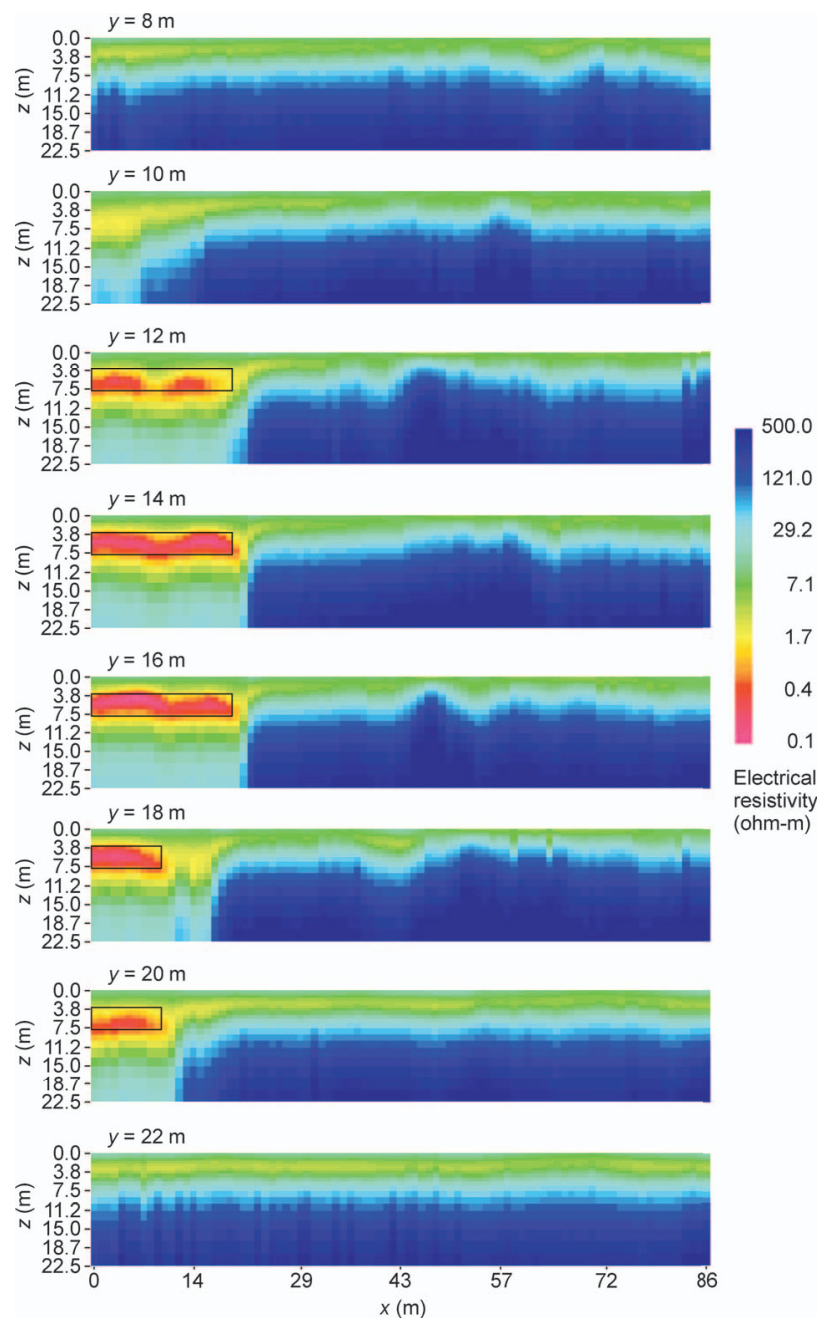

Figure 11. Final image of the subsoil obtained by applying the EM1DFM 1D inversion code to the quadrature components filtered two times using the preinversion data-smoothing filter defined by equation 2 with $N=3$ and $A=2$. Approximate boundaries of the waste deposit along each line, determined from excavations performed later, also are indicated.

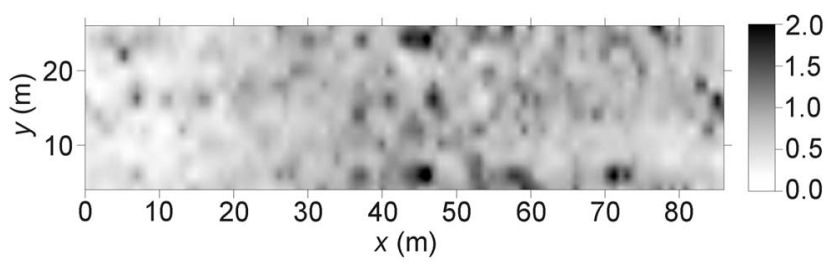

Figure 12. A 2D plot of the misfit corresponding to the final model in Figure 11. one extending from $x=0$ to $20 \mathrm{~m}$ and $y=11$ to $17 \mathrm{~m}$, approximately, and the other extending from $x=0$ to $10 \mathrm{~m}$ and $y=17$ to $21 \mathrm{~m}$. The approximate limits of these deposits are indicated in Figure 11 for comparison. The modeling results and field findings agree very well.

\section{CONCLUSIONS}

We have presented the results of the numerical modeling of SLEM data acquired at an agrochemical plant. The goal of the study was to obtain useful information before initiating remediation work. By direct observation of data, we identified an area with anomalously increased responses that could be caused by the presence of buried waste or contamination. Next we obtained an electrical image of the subsoil at each line in that area by applying a 1D inversion method. The results confirmed that $1 \mathrm{D}$ inversion methods effectively detect extended conductive anomalies and provide a rapid way to obtain an approximate characterization of their electrical structure. In our case, even the top and the bottom of the more conductive zone were delimited roughly. Data inversion required only 15-20 minutes on a PC. It is true that in certain situations, an adequate characterization of target structures or even their detection can be achieved only by applying $2 \mathrm{D}$ or $3 \mathrm{D}$ inversion codes, but this requires much longer computing times, especially when large amounts of data must be processed. In many other cases, such as the one considered here, acceptable results can be obtained much faster from 1D inversions.

The subsoil models obtained along each line varied gradually with depth but exhibited unrealistic conductivity jumps in the horizontal direction. This problem, which deteriorated the visualization of the anomaly, is very common in models built by joining $1 \mathrm{D}$ inversion results because 1D inversion codes generally apply smoothness constraints in the vertical direction but do not apply any kind of correlation between the results obtained at neighboring sounding points. So lateral variations are freely allowed. Some correspond to real changes in the subsoil structure, but others are spurious artifacts associated with data noise and variations in the misfits attained from point to point. The presence of $2 \mathrm{D}$ or $3 \mathrm{D}$ effects can increase lateral variability further.

To overcome these difficulties, we designed and applied two lateral smoothing filters. One correlates the data from neighboring points (considering the two directions parallel and perpendicular to survey lines) prior to inversions, and the other correlates, in a similar manner, the 1D inverse models obtained after inverting the original data. Both proved to be very effective for reducing spurious lateral variations in these kinds of models. They are not rigid. On the contrary, they can be adapted to the requirements of each particular situation and are highly stable because they can be applied several times before significantly oversmoothing the anomalies. In addition, they are extremely fast; each filtering of our data set took less than $5 \mathrm{~s}$, running on a common PC. As opposed to laterally constrained inversion schemes, the filtering is completely independent of the 1D inversion method used. In our case, each filter greatly improved the subsoil models obtained for all of the lines, especially those corresponding to lines crossing near or over the anomaly. Consolidating those results, we finally obtained a high-quality $3 \mathrm{D}$ visualization of the target.

Remediation excavations made later at the site confirmed the results of our numerical modeling scheme. Hundreds of barrels containing production materials were found in the anomalous zone, buried near the predicted depths. 


\section{ACKNOWLEDGMENTS}

This work was supported partially by Consejo Nacional de Investigaciones Científicas y Técnicas (CONICET) and Agencia Nacional de Promoción Científica y Tecnológica (ANPCyT). We especially thank Colin G. Farquharson, Douglas W. Oldenburg, and Partha S. Routh for providing a complimentary copy of the EM1DFM code.

\section{REFERENCES}

Ao, C. O., H. Braunisch, K. O’Neill, and J. A. Kong, 2002, Quasi-magnetostatic solution for a conducting and permeable spheroid with arbitrary excitation: IEEE Transactions on Geoscience and Remote Sensing, 40, 887-897.

Auken, E., A. V. Christiansen, B. H. Jacobsen, N. Foged, and K. I. Sørensen, 2005, Piecewise 1D laterally constrained inversion of resistivity data: Geophysical Prospecting, 53, 497-506.

Auken, E., L. Pellerin, N. B. Christensen, and K. Sørensen, 2006, A survey of current trends in near-surface electrical and electromagnetic methods: Geophysics, 71, no. 5, G249-G260.

Bell, T.H., B. J. Barrow, and J. T. Miller, 2001, Subsurface discrimination using electromagnetic induction sensors: IEEE Transactions on Geoscience and Remote Sensing, 39, 1286-1293.

Butler, D. K., 2004, Report on a workshop on electromagnetic induction methods for UXO detection and discrimination: The Leading Edge, 23, 766-770.

Eigenberg, R. A., and J. A. Nienaber, 2003, Electromagnetic induction methods applied to an abandoned manure handling site to determine nutrient buildup: Journal of Environmental Quality, 32, 1837-1843.

Farquharson, C. G., D. W. Oldenburgh, and P. S. Routh, 2003, Simultaneous 1D inversion of loop-loop electromagnetic data for magnetic susceptibility and electrical conductivity: Geophysics, 68, 1857-1869.

Geng, N., C. E. Baum, and L. Carin, 1999, On the low-frequency natural response of conducting and permeable targets: IEEE Transactions on Geoscience and Remote Sensing, 37, 347-359.

Haber, E., U. M. Ascher, and D. W. Oldenburg, 2004, Inversion of 3D electromagnetic data in frequency and time domain using an inexact all-atonce approach: Geophysics, 69, 1216-1228.

Hendrickx, J. M. H., B. Borchers, D. L. Corwin, S. M. Lesch, A. C. Hilgendorf, and J. Schlue, 2002, Inversion of soil conductivity profiles from electromagnetic induction measurements: Theory and experimental verification: Soil Science Society of America Journal, 66, 673-685.

Huang, H., and I. J. Won, 2000, Conductivity and susceptibility mapping using broadband electromagnetic sensors: Journal of Environmental and Engineering Geophysics, 5, 31-41.

Lascano, E., P. Martinelli, and A. Osella, 2006, EMI data from an archaeological resistive target revisited: Near Surface Geophysics, 4, 395-400.

Martinelli, P., A. Osella, and E. Lascano, 2006, Modeling broadband electromagnetic induction responses of 2-D multilayered structures: IEEE Transactions on Geoscience and Remote Sensing, 44, 2454-2460.

McNeill, J. D., 1980, Electromagnetic terrain conductivity measurements at low induction numbers: Geonics Limited Technical Note TN-6, http:// www.geonics.com/pdfs/technicalnotes/tn6.pdf.

McNeill, J. D., and M. Bosnar, 1999, Application of dipole-dipole electro- magnetic systems for geological depth sounding: Geonics Limited Technical Note TN-31, http://www.geonics.com/pdfs/technicalnotes/tn31.pdf.

Miller, J. T., T. H. Bell, J. Soukup, and D. Keiswetter, 2001, Simple phenomenological models for wideband frequency-domain electromagnetic induction: IEEE Transactions on Geoscience and Remote Sensing, 39, 1294-1298.

Mitsuhata, Y., 2000, 2D electromagnetic modeling by finite-element method with a dipole source and topography: Geophysics, 65, 465-475.

Mitsuhata, Y., T. Uchida, and H. Amano, 2002, 2.5-D inversion of frequencydomain electromagnetic data generated by a grounded-wire source: Geophysics, 67, 1753-1768.

Monteiro Santos, F. A., 2004, 1-D laterally constrained inversion of EM34 profiling data: Journal of Applied Geophysics, 56, 123-134.

Newman, G. A., and D. L. Alumbaugh, 1995, Frequency-domain modelling of airborne electromagnetic responses using staggered finite differences: Geophysical Prospecting, 43, 1021-1042.

-, 1997, Three-dimensional massively parallel electromagnetic inversion - I: Theory: Geophysical Journal International, 128, 345-354.

Osella, A., M. de la Vega, and E. Lascano, 2005, 3D electrical imaging of an archaeological site using electrical and electromagnetic methods: Geophysics, 70, no. 4, G101-G107.

Pérez-Flores, M. A., S. Méndez-Delgado, and E. Gómez-Treviño, 2001, Imaging low-frequency and DC electromagnetic fields using a simple linear approximation: Geophysics, 66, 1067-1081.

Sasaki, Y., 2001, Full 3-D inversion of electromagnetic data on PC: Journal of Applied Geophysics, 46, 45-54.

Sasaki, Y., and M. A. Meju, 2006, A multidimensional horizontal-loop controlled-source electromagnetic inversion method and its use to characterize heterogeneity in aquiferous fractured crystalline rocks: Geophysical Journal International, 166, 59-66.

Shubitidze, F., K. O’Neill, S. A. Haider, K. Sun, and K. D. Paulsen, 2002, Application of the method of auxiliary sources to the wide-band electromagnetic induction problem: IEEE Transactions on Geoscience and Remote Sensing, 40, 928-942.

Sun, K., K. O’Neill, F. Shubitidze, I. Shamatava, and K. D. Paulsen, 2004, Theoretical analysis and range of validity of TSA formulation for application to UXO discrimination: IEEE Transactions on Geoscience and Remote Sensing, 42, 1871-1881.

Tezkan, B., 1999, A review of environmental applications of quasi-stationary electromagnetic techniques: Surveys in Geophysics, 20, 279-308.

Unsworth, M. J., B. J. Travis, and A. D. Chave, 1993, Electromagnetic induction by a finite electric dipole source over a 2-D earth: Geophysics, $\mathbf{5 8}$ $198-214$.

Witten, A. J., G. Calbert, B. Witten, and T. Levy, 2003, Magnetic and electromagnetic induction studies at archaeological sites in southwestern Jordan Journal of Environmental and Engineering Geophysics, 8, 209-215.

Won, I. J., 2003, Small frequency-domain electromagnetic induction sensors: The Leading Edge, 22, 320-322.

Won, I. J., D. A. Keiswetter, and T. H. Bell, 2001, Electromagnetic induction spectroscopy for clearing landmines: IEEE Transactions on Geoscience and Remote Sensing, 39, 703-709.

Won, I. J., D. A. Keiswetter, G. R. A. Fields, and L. C. Sutton, 1996, GEM-2: A new multifrequency electromagnetic sensor: Journal of Environmental and Engineering Geophysics, 1, 129-138.

Won, I. J., D. A. Keiswetter, and E. Novikova, 1998, Electromagnetic induction spectroscopy: Journal of Environmental and Engineering Geophysics, 3, 27-40. 